\title{
Design of Calibration platform for DC current transformer based on LabVIEW
}

\author{
Zhiyuan Xie \& Sisi Han \\ Department of Electronic and Communication Engineering, North China Electric Power University, Baoding, \\ China \\ Lichong Wang \\ Hebei Shenke Electronics Co., Ltd., Shijiazhuang, China
}

\begin{abstract}
According to the verification requirement of DC current transformer, a multi channel DC transformer calibration platform based on virtual instrument is designed and built. Using Laboratory Virtual Instrument Engineering Workbench (LabVIEW) programming, it can play a flexible, customizable, powerful data processing and analysis functions. The verification platform includes three parts, the multi-channel data acquisition, the LabVIEW processing platform and the RS485 bus. Data acquisition part uses 16 channels synchro-nous sampling. The experimental results show that the method based on the virtual instrument calibration method is convenient and effective, and it can improve the efficiency and the automation level of the mutual verification.
\end{abstract}

KEYWORD: DC transformer; LabVIEW; Data acquisition; Multi-channel; RS485

\section{INTRODUCTION}

DC transformer is used to measure the DC current and used for DC rectification system to do current feedback control of the electrical components. With the development and application of DC power transmission is becoming more and more popular, we need a large of DC equipment and tools, and DC transformer as a key device for DC measurement and protection must be used, the current market demand increased year by year.

In order to ensure the normal and safe operation of the DC measurement and protection system, the DC transformer calibration is a indispensable work (Lin Xiao-mei, Sun Shen-yan, 1993).small direct current transformer is not to be checked accurately, and each of these calibration system can only check the mutual inductor, the efficiency is relatively low. Therefore, it is necessary to design a calibration platform for DC current transformer.

In this paper, the virtual instrument technology is introduced into the calibration platform of DC transformer, and the LabVIEW is used as the development tool. LabVIEW is a graphical programming software, it is widely used in industry, academia and laboratory studies, is seen as the standard data acquisition and instrument control software (Cheng Lei, 2009). When using the graphical programming language programming, the main use of flow chart or diagram to complete the various functions of the instrument, we don't need to write code, to a large extent to avoid the procedure code compilation and hardware circuit design, so as to shorten the development cycle.

\section{SYSTEM DESIGN}

The block diagram of the DC transformer calibration system is shown in Figure 1 . The verification platform mainly consists of 2 parts, the host computer and the slave computer. The virtual instrument is the core of the control system, which is composed of host computer, transformer calibrator, data acquisition card, RS485 bus (YANG Zi-qiang, 2002) are composed of slave computer, each of the transformer calibrator as a terminal. The host computer uses the RS232 serial port, through the RS232/485 converter connected to the 485 bus, the terminal using the UART serial port, through the RS485/UART converter and the 485 bus connection. The main process: data acquisition card real-time collect 15 road transformer and 1 reference voltage value, the collected data by transmitting to computer LabVIEW, based on virtual instrument (VI) is the core of the control system of the data for analysis and processing, through the recognition device address and the corresponding transformer communication, 15 road transformer terminal receiving command of virtual instrument, command parsing, automatic fufil calibration function. 


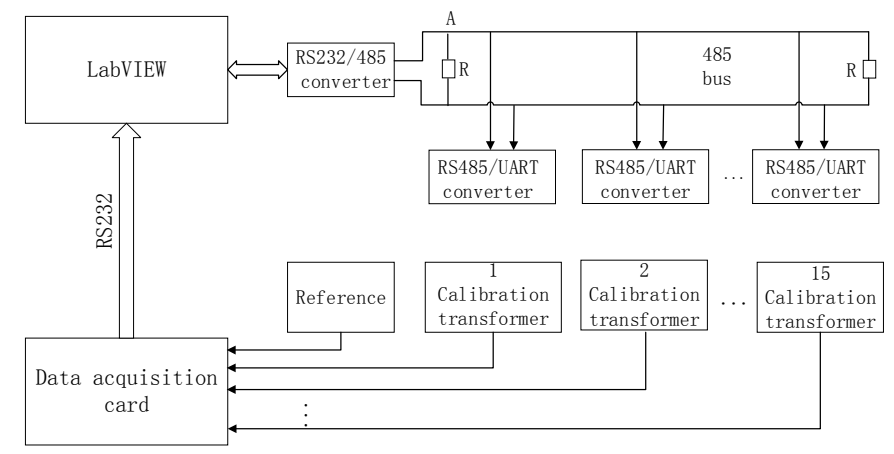

Figure 1. System structure diagram

\section{HARDWARE DESIGN}

The hardware design of the calibration platform is mainly composed of data acquisition module and RS485 bus interface. The data acquisition module adopts the USB1901 data acquisition card of the Ling Hua technology, and the communication interface adopts the 485 bus.

\subsection{Data acquisition module}

The data acquisition module collects external signal, and handles the input analog signal, and then input to the computer as the core of virtual instrument in analyzing and processing (Li Wei-xuan, 2005).

In the verification platform, the collect data is multi-channel analog voltage, and require rapid and accurate data collection. ADLINK technology USB multi function data acquisition module USB-1901 is non Ni Company into production of plug and play data acquisition card, the acquisition card in the LabVIEW platform can directly drive. It has 16 bit high resolution, sampling of the analog input rate reached the highest $250 \mathrm{kS} / \mathrm{s}$ and provides high performance of the A / D and D / A conversion, and simulation output update frequency of $1 \mathrm{MS} / \mathrm{s}$, fully meet the needs of the verification platform.

\section{SOFTWARE DESIGN}

System software design includes virtual instrument program design. The design based on LabVIEW 2013 development, in the startup window, we select "new VI", enter the virtual instrument VI programming environment. Including the front panel design, block diagram design.

\subsection{Front panel design}

The front panel is the user interface of the VI program. It is an interactive input and output port of the VI program, It is usually used to create the front panel with the input control and the display control. Input control and output control are respectively used to simulate the input and output device of the instrument. The former provides data for the block diagram of the VI, The latter is used to display the program block diagram to obtain or generate the data (Chen Shu-xue, 2001).

The main interface includes serial port parameter configuration, operation control buttons, real-time data display and real-time waveform display.

Serial communication parameters setting for the host computer and RS232 communication parameters (serial number, baud rate, etc.). Operation control button is used to start or stop the data acquisition card data acquisition, data storage and data playback. The data display is used to display the real-time data of each acquisition channel, and the real-time waveform is used to display the real-time voltage value of each acquisition channel.

\subsection{Program block diagram}

The block diagram is a collection of graphical source code, which determines the operation mode of VI. Input controls and indicators in the diagram has a target endpoint-one correspondence. The wires put the input control and indicator terminals connected to each other with the VI and functions, they are data channel ports.

\subsection{Check the programming design}

The control circuit board of the DC current transformer is mainly controlled by ATmeg16. TLC5615 is a serial 10 bit D/A conversion chip. The serial input of 10 bits data can be completed by 3 serial bus. The DAC circuit using ATmeg16 MCU general I/O port and TLC5615 form as shown in Figure 2. Respectively with PA1、PA2 simulates clock SCLK and $\overline{C S}$, the binary number to be converted from the PA0 output to TLC5615 data input DIN [Li Hua-gui, 1999].

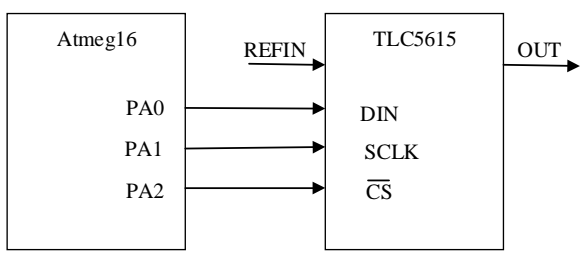

Figure 2. Diagram of Hardware connection

The output voltage of the TLC5615 chip is: $V_{\text {out }}=2 \times V_{\text {REFIN }} \times N / 1024$

Among them, VREFIN is the reference voltage, $N$ is the input binary number.

The output voltage calibration of the DC current transformer is to adjust the input binary number in the D/A chip. The N/1024 is regarded as a coefficient $K$, and the calibration of the DC current transformer is the adjustment of the coefficient $\mathrm{K}$.

The LabVIEW can quickly write user interface, it also is convenient to set and debug of each input and output parameters, the result of the program directly, 
but in the aspect of numerical analysis and algorithm is not perfect, so for some complex data processing applications, LabVIEW has some deficiencies. And MATLAB has a powerful numerical calculation, a lot of tools. So combine the reliable algorithm with graphical programming capabilities, the mixed programming can achieve a very good effect [WEI Liang, 2003]. Ni Company provides a way of calling MATLAB, namely matlab script node, matlab scripts can write directly in the MATLAB script node, when the VI is running the MATLAB will automatically start and execute script in the contents of the program [Wang L, 2002]. The node can add the input and output to the shortcut menu. As shown in Figure 3, that is to add the script program and input, the output of the script node (Li Ning, 2003, LI Xiao-yan, 2008).The difference between the DC transformer and the reference voltage is input to the Script MATLAB node, The MATLAB to determine the difference, the size of the $\mathrm{K}$ value of each channel to make the corresponding adjustment. Through this programming method, the output of the coefficient $\mathrm{K}$ is realized quickly. Before switching to the front panel, the voltage of the collected DC current transformer can be observed to be adjusted to the same as the reference voltage.

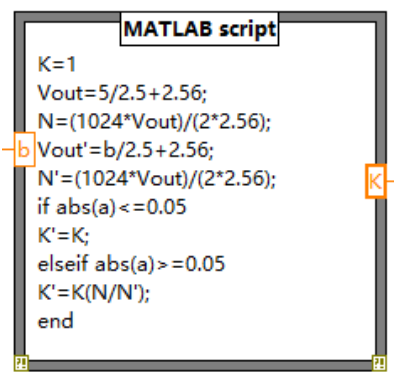

Figure 3. MATLAB Script node

\subsection{Modbus protocol selection}

Modbus communication protocol has ASCII and RTU two message frame format. The verification platform uses RTU transmission mode to communicate, in the message every eight byte contains two 4-byte hexadecimal characters. The advantages of this approach are mainly in the same baud rate, transmit the same information require less bits .The Modbus transmission format in RTU mode is shown in Table 1.

Modbus communication protocol using masterslave structure that allows a host device and multiple slave device communication, only the main equipment can from device sends communication instruction, from the device according to the main equipment of communication instructions to make corresponding response. In the verification platform, the main equipment is the computer, slave device is DC current transformer. Device address, this refers to the address value of the DC transformer, It indicates that LabVIEW will specify and from a communication device unique identifier. Function code is used to identify the function of the communication frame. Make the device address of the first transformer terminal is 01 , according to the Modbus protocol standard, the function code of the sending command is 06 , the check coefficient $\mathrm{K}$ is converted to sixteen binary data code to send from the device. The last two bytes of the sending instruction are the CRC verification code, which is used to implement the error control of the Modbus protocol transmission of the master slave device.

Table 1. RTU message frame

\begin{tabular}{cc}
\hline Start bit & T1-T2-T3-T4 \\
\hline Device address & 8bit \\
Function code & 8bit \\
Data & N 8bit \\
CRC checksum & $16 \mathrm{bit}$ \\
Terminator & T1-T2-T3-T4 \\
\hline
\end{tabular}

\section{RESULTS ANALYSIS}

In order to verify the calibration accuracy of the device, the measurement and comparison of the DC transformer after calibration are carried out. The experimental results are as follows.

Table 2. Results of Experimental display ( $\mathrm{I}=0 \mathrm{~mA})$

\begin{tabular}{llllll}
\hline $\begin{array}{l}\text { Channel } \\
\text { number }\end{array}$ & $\begin{array}{l}\text { Error } \\
\text { value }\end{array}$ & $\begin{array}{l}\text { Channel } \\
\text { number }\end{array}$ & $\begin{array}{l}\text { Error } \\
\text { value }\end{array}$ & $\begin{array}{l}\text { Channel } \\
\text { number }\end{array}$ & $\begin{array}{l}\text { Error } \\
\text { value }\end{array}$ \\
\hline 1 & 0 & 6 & -0.0156 & 11 & -0.0298 \\
2 & -0.0475 & 7 & -0.0164 & 12 & -0.0037 \\
3 & -0.0158 & 8 & -0.0166 & 13 & 0.0033 \\
4 & 0 & 9 & -0.0159 & 14 & 0.0280 \\
5 & 0 & 10 & 0.0154 & 15 & 0.0005 \\
\hline
\end{tabular}

Table 3. Results of Experimental display ( $\mathrm{I}=2 \mathrm{~mA})$

\begin{tabular}{cccccc}
\hline $\begin{array}{c}\text { Channel } \\
\text { number }\end{array}$ & $\begin{array}{c}\text { Error } \\
\text { value }\end{array}$ & $\begin{array}{c}\text { Channel } \\
\text { number }\end{array}$ & $\begin{array}{c}\text { Error } \\
\text { value }\end{array}$ & $\begin{array}{c}\text { Channel } \\
\text { number }\end{array}$ & $\begin{array}{c}\text { Error } \\
\text { value }\end{array}$ \\
\hline 1 & 0.0096 & 6 & -0.0189 & 11 & 0.0049 \\
2 & 0.0317 & 7 & -0.0034 & 12 & 0.0134 \\
3 & 0.0127 & 8 & 0.0066 & 13 & 0.0126 \\
4 & -0.0096 & 9 & 0.0032 & 14 & -0.0034 \\
5 & 0.0033 & 10 & -0.0186 & 15 & 0.0224 \\
\hline
\end{tabular}

Table 4. Results of Experimental display ( $\mathrm{I}=5 \mathrm{~mA})$

\begin{tabular}{cccccc}
\hline $\begin{array}{c}\text { Channel } \\
\text { number }\end{array}$ & $\begin{array}{c}\text { Error } \\
\text { value }\end{array}$ & $\begin{array}{c}\text { Channel } \\
\text { number }\end{array}$ & $\begin{array}{c}\text { Error } \\
\text { value }\end{array}$ & $\begin{array}{c}\text { Channel } \\
\text { number }\end{array}$ & $\begin{array}{c}\text { Error } \\
\text { value }\end{array}$ \\
\hline 1 & 0.0160 & 6 & 0.0157 & 11 & -0.0019 \\
2 & 0.0317 & 7 & 0.0165 & 12 & 0.0038 \\
3 & 0 & 8 & 0 & 13 & -0.0111 \\
4 & 0 & 9 & 0.0160 & 14 & 0.0011 \\
5 & 0 & 10 & 0 & 15 & 0.0438 \\
\hline
\end{tabular}


Table 5. Results of Experimental display ( $\mathrm{I}=10 \mathrm{~mA})$

\begin{tabular}{cccccc}
\hline $\begin{array}{c}\text { Channel } \\
\text { number }\end{array}$ & $\begin{array}{c}\text { Error } \\
\text { value }\end{array}$ & $\begin{array}{c}\text { Channel } \\
\text { number }\end{array}$ & $\begin{array}{c}\text { Error } \\
\text { value }\end{array}$ & $\begin{array}{c}\text { Channel } \\
\text { number }\end{array}$ & $\begin{array}{c}\text { Error } \\
\text { value }\end{array}$ \\
\hline 1 & 0 & 6 & 0.0495 & 11 & 0.0209 \\
2 & 0.1111 & 7 & 0 & 12 & 0.0173 \\
3 & -0.0160 & 8 & 0.0480 & 13 & -0.0053 \\
4 & -0.0166 & 9 & 0.0154 & 14 & 0.0033 \\
5 & 0 & 10 & -0.0065 & 15 & 0.0545 \\
\hline
\end{tabular}

Table 6. Results of Experimental display (I=20mA)

\begin{tabular}{cccccc}
\hline $\begin{array}{c}\text { Channel } \\
\text { number }\end{array}$ & $\begin{array}{c}\text { Error } \\
\text { value }\end{array}$ & $\begin{array}{c}\text { Channel } \\
\text { number }\end{array}$ & $\begin{array}{c}\text { Error } \\
\text { value }\end{array}$ & $\begin{array}{c}\text { Channel } \\
\text { number }\end{array}$ & $\begin{array}{c}\text { Error } \\
\text { value }\end{array}$ \\
\hline 1 & 0 & 6 & 0.1155 & 11 & -0.0206 \\
2 & 0.1587 & 7 & 0.0320 & 12 & -0.0137 \\
3 & 0 & 8 & 0.0619 & 13 & -0.0053 \\
4 & -0.0166 & 9 & 0.0361 & 14 & -0.0022 \\
5 & 0 & 10 & 0.0413 & 15 & 0.0455 \\
\hline
\end{tabular}

From the experimental results, after a check of the verification platform, the error value is less than 0.05 . Therefore, this verification system to DC transformer to carry out calibration work is feasible.

\section{CONCLUSION}

Counted in check in this paper, using the virtual instrument technology in the computer implementation of the DC current transformer verification platform design, it mainly has the following characteristics: (1) In the structure, the LabVIEW platform is PC machine to achieve the instrument calibration system function mainly through installed in the machine on the LabVIEW programming environment to achieve rapid; (2) In the function, the verification platform verified at the same time multiplex transformer not only can collect data and calculated data, send instructions, but also real-time data display, automatic storage; (3) In the operation, LabVIEW makes the platform operation is greatly simplified, and all operations are in the front panel. To provide a reliable and convenient tool for successful design of the verification platform for verification of DC transformer.

\section{REFERENCE}

Cheng Lei. The on-line calibration system of High-Voltage current transformer based on virtual instrument [D]. Huazhong University of Science and Technology.2009.

Chen Shu-xue, Liu Xuan.LabVIEW collection [M]. Beijing:

Electronic Industry Press, 2001.

Lin Xiao-mei,Sun Shen-yan. Analysis of working principle of DC transformer and intelligent DC current transformer[J]. Electrical measurement \& instrumentation,1993,(7):9-14.

Li Wei-xuan,Huang Jian-xin.The Driving Methods for Common DAQ Card and Data Acquisition Based on LabVIEW[J].Electronics Quality,2005,(7):14-16,13.
Li Hua-gui, Wang Yun-peng. Serial 10 digit / analog converter TLC5615 and its application in the single chip microcomputer [J]. foreign electronic components.1999, (5): 29-31.

Li Ning,Zhang Yuan-pei,Zhu Li-jun. Using MATLAB Script in LabVIEW[J].Instrument Standardization \&Metrology, 2003, (5): 17-19.

LI Xiao-yan, YANG Jun-yu, ZHAO Jia .The realization of mxed programming with Matlab and LabVIEW[J]. Electronic Instrumentation Customer,2008,15(2):101-102.

National Instruments Corportion.LabVIEW User Manual [M].Texas:National Instruments,2003.

The portable USB interface data acquisition module [J]. Automation application, 2011, (11): 38.

WEI Liang. Hybrid programming with LabVIEW and MATLAB/SIMLULNK and its application in control system[J]. Electronic Instrumentation Customer, 2013 (1): 95-96.

Wang L. The Application of LabVIEW inData Acquisition System of Solar Absorption Refrigerator [C]. HongKong, 2012 International Conference on Future Energy, Environmentand Materials, 2012: 1496-1502.

XIE Qi.Realization of Modbus RTU Communication Protocol Based on LabVIEW[J].Coal Mine Machinery,2006,27(12): 95-97.

YANG Fang.Design and Realization on Stepping Motor Control SystemBased on Master-Slave Distributive SerialMultiComputer Communication[J].Journal of Shanghai University of Electric Power,2013,29(3): 234-237.

YANG Zi-qiang,REN Feng,AI Xin.Current transformer calibrator based on virtual instrument technology [J]. Electrical Measurement \& Instrumentation, 2002, 39(12): 33-35. 